\title{
Cationic Ordering and Magnetic Properties of Re-Based Double Perovskite Oxides
}

\author{
Si-Young Choi ${ }^{1}$, Minseok Choi ${ }^{1}$, Sung-Dae Kim ${ }^{1}$, Hyung Jeen Jeen ${ }^{2}$, and Young-Mok Rhyim ${ }^{1}$ \\ 1. Korea Institute of Materials Science, Changwon 641-831, Korea \\ 2. Department of Physics, Pusan National University, Busan 609-735, Korea
}

$\mathrm{Sr}_{2} \mathrm{FeReO}_{6}$ (SFRO) and $\mathrm{Sr}_{2} \mathrm{CrReO}_{6}$ (SCRO) are ferrimagnetic with tetragonal structure (space group, $\mathrm{I} / \mathrm{m}$ ) and their magnetic properties are strongly influenced by $\mathrm{Fe} / \mathrm{Re}$ and $\mathrm{Cr} / \mathrm{Fe}$ cationic ordering [1]. Our aim is to fully understand the correlation between cationic ordering and magnetization in SFRO and SCRO. To this end, we performed a comprehensive study on cationic ordering and magnetic properties in SFRO and SCRO through a series of experiments by combining theoretical calculations. A variety of the samples were synthesized and characterized in terms of microstructure and magnetic properties by varying an amount of excessive Re during sample growth.

SFRO and SCRO powders were prepared via conventional solid state reaction. Since Rhenium has the volatile nature, amount of Re-excess is controlled in order to create antisite defects. All the mixed powders were pressed into disks and contact between pellets and crucible is minimized in order to avoid Re loss. The pellets were calcined at $1000{ }^{\circ} \mathrm{C}$ for $10 \mathrm{hr}$ under Ar atmosphere (nominal purity 99.99\%). The prepared powders were sintered by spark plasma sintering (SPS) at $1150 \circ \mathrm{C}$ for 10 min under the 65 MPa in Ar atmosphere. Their microstructural and morphological properties were characterized, and distribution of antisite defects was checked by high-angle annular dark-field scanning transmission electron microscopy (HADDF-STEM). The magnetic properties were measured via vibrating sample magnetometer (VSM) driven up to a magnetic field of $9 \mathrm{~T}$. The calculations were performed using the projector augmented-wave method and the Perdew-Burke-Ernzerhof (PBE)-GGA exchange-correlation functional with a Hubbard-U correction $(G G A+U)$ as implemented in the VASP code. For defect simulations, the 320-atom supercells were used. The wavefunctions were expanded in a plane-wave basis set with an energy cutoff of $400 \mathrm{eV}$, and integrations over the Brillouin zone were carried out using the $2 \times 2 \times 2 \mathrm{k}$-point mesh. The atomic coordinates were relaxed until the force acting on each atom was reduced to less than $0.02 \mathrm{eV} / \AA$.

The series of experiments, the formation of antisite defects, which determines the net magnetization of SFRO and SCRO, strongly correlates with the amount of added Re (fig. 1). Antisite defects are clustered in a type of antiphase-boundary-like microstructure in SFRO whereas they are spatially well distributed in the whole SCRO samples, as shown in fig. 2. The cluster-type antisite defects in SFRO deteriorated net magnetization than that from the scattered case in SCRO. Through first-principles density-functional calculations, we suggest that the differences can be explained in terms of defect formation energy and binding energy, determined by interaction between antisite defects. Our calculations show that antisite defects in SFRO are likely to form and be clustered due to attractive interaction between them, but in SCRO they favor to be spatially scattered. Our findings provide the fundamental understanding of Rebased double perovskite oxides and pave the way to experimentalists to get better quality samples.

Acknowledgement

This work was supported by the Global Frontier R \& D Program on Center for Hybrid Interface Materials (HIM) (GFHIM-2013M3A6B1078872).

Reference 
[1] B. Blamire, J. L. MacManus-Driscoll, N. D. Mathur and Z. H. Barber, Adv. Mater. 21 (2009), p. 3827.
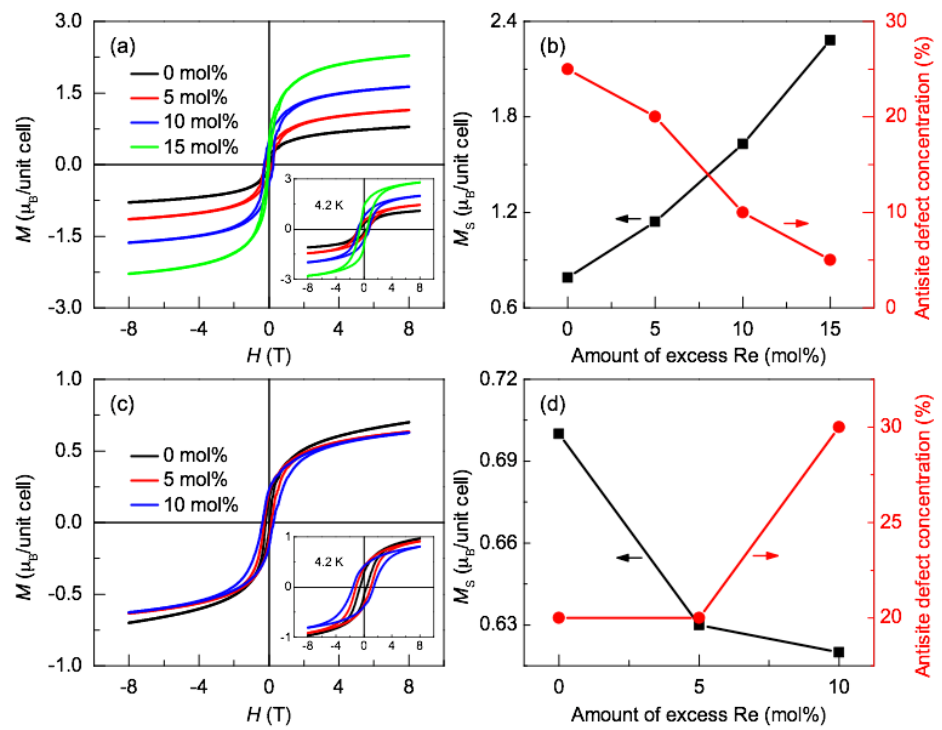

Figure 1. (a, c) Magnetization (M) - magnetic field (H) hysteresis loop and (b, d) correalated values of the magnetic saturation and the antisite defect concentration at $300 \mathrm{~K}$. The amount of excess Re is (a, b) SFRO-xRe $(0 \mathrm{~mol} \% \leq \mathrm{x} \leq 15 \mathrm{~mol} \%)$ and (c, d) SCRO-xRe $(0 \mathrm{~mol} \% \leq \mathrm{x} \leq 10 \mathrm{~mol} \%)$. In the inset of (a) and (c), M-H loop measured at $4.2 \mathrm{~K}$ is presented for comparison.
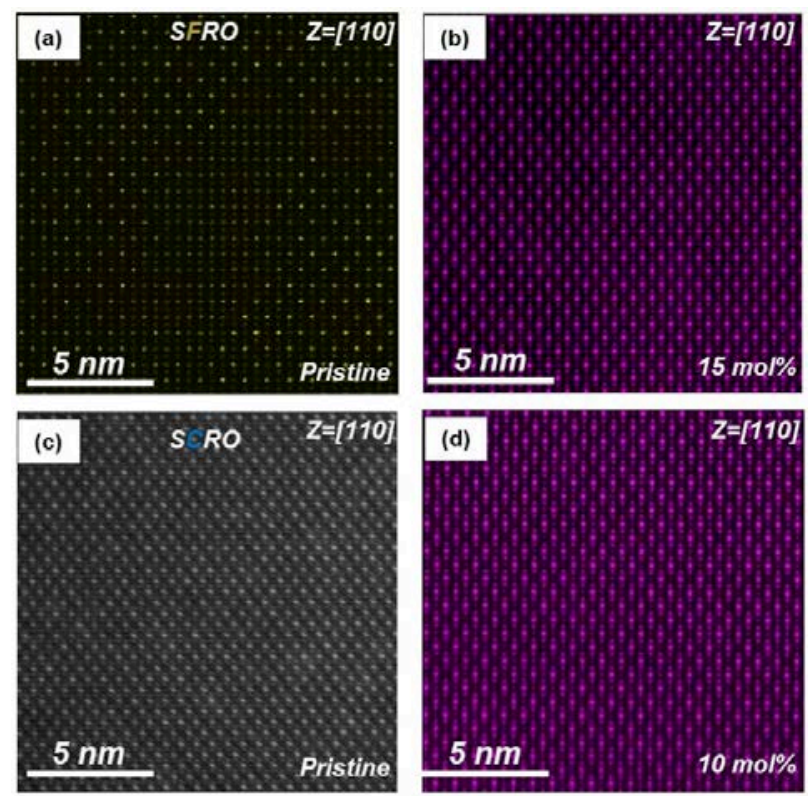

Figure 2. HADDF-STEM image of (a) defective pristine SFRO and (b) well-ordered SFRO by 15 mol\% Re-excess; (c) pristine SCRO and (d) 10 mol\% Re-excess SCRO. Note that (c) and (d) samples hold the significant amount of antisite defects, 25\%. 